\title{
O Papel do Estado e Cultura Política na Argentina e no Brasil
}

\author{
Daniel Jaime Capistrano \\ Henrique Carlos de O. Castro \\ Centro de Pesquisa e Pós-Graduação sobre as Américas \\ Universidade de Brasília
}

\begin{abstract}
Resumo: A opinião e a orientação de argentinos e brasileiros em relação à política e ao Estado têm mudado nos últimos vinte anos? Transformações sociais ocorridas nesse período sugerem que a cultura política nesses países experimentou alterações importantes. O presente artigo aborda esse tema com ênfase no apoio público à intervenção do Estado na economia. Os resultados da pesquisa corroboram a lógica de transformação na cultura política, mas apresentam um padrão de valorização da ação do Estado que não é considerado no debate atual. Esse padrão pode ser explicado por aspectos históricos, sociais e políticos específicos dos países latinoamericanos.
\end{abstract}

Palavras-chave: cultura política; mudança política; Estado; Brasil; Argentina

\begin{abstract}
Have the opinions and guidance of Argentines and Brazilians on politics and the state changed over the last twenty years? Several social changes during this period suggest that the political culture of these countries has also experienced a transformation. This work addresses this issue by emphasizing the social support for state intervention in the economy. The research results confirm the logic of change in political culture; however it shows a pattern of increasing emphasis on state action, which is not considered in the current debate. This pattern could be explained by historical, social and political characteristics of Latin American countries.
\end{abstract}

Keywords: political culture; political change; State; Brazil; Argentina 


\section{Introdução}

Uma dimensão fundamental do processo de modernização é a constante transformação do papel do Estado nas diversas sociedades. Do laissez-faire ao desenvolvimentismo, do Consenso de Washington à crise financeira de 2008, o Estado assumiu diversas configurações e papéis de acordo com a ideologia dominante, a tradição política de cada sociedade e as conjunturas sociais e econômicas de cada país. Contudo, nas últimas décadas, pouco se avançou em relação a uma análise específica do apoio popular aos diversos modelos de Estado na América Latina. O presente artigo, realizado a partir dos dados da Pesquisa Mundial de Valores (World Values Survey - WVS ${ }^{1}$ ), procura identificar a dinâmica de valorização do Estado entre argentinos e brasileiros.

A discussão mais rica em relação a essa dimensão é feita de maneira generalizada por meio dos trabalhos sobre mudança na cultura política cujo debatedor mais conhecido é Ronald Inglehart. Contudo, existe uma lacuna nessa literatura que não incorpora os contextos histórico, social e político das nações como fatores determinantes da formação e mudança da cultura política ${ }^{2}$. A incorporação de elementos ligados a essas questões traz benefícios não somente ao debate sobre os resultados de pesquisa como, principalmente, à operacionalização mais apurada do conceito de "cultura política".

Dessa forma, antes de dar início à descrição da pesquisa realizada, a parte inicial desse artigo está dedicada a uma análise crítica do referencial teórico da cultura política levantando algumas questões essenciais para que essa abordagem fosse adotada como base para a pesquisa descrita na segunda parte deste artigo.

A proposta de alguns estudos mais recentes de cultura política (INGLEHART, 1997; INGLEHART e WELZEL, 2005) é investigar uma determinada síndrome de mudança cultural que enfatiza a autonomia do indivíduo e o questionamento da autoridade do Estado em todos os países do globo. Estudos anteriores (CASTRO, 2000; CAPISTRANO, 2008; CASTRO, 2008) apontam que esse viés é fruto de uma determinada visão de modernização e de uma conceituação de cultura política que enfatiza aspectos comuns desse processo em todas as sociedades negligenciando características peculiares da formação histórica, social e política de cada caso.

Ou seja, para se utilizar a perspectiva de análise política proposta por esses autores são necessárias duas ressalvas: i) a operacionalização do conceito de cultura política tende a valorizar aspectos de uma determinada sociedade quando são criados para analisá-la e; ii) a força motriz da transformação da cultura política, a modernização, não é um processo homogêneo e se torna impreciso quando

\footnotetext{
${ }^{1}$ A pesquisa WVS é realizada em cerca de 100 países, com levantamentos de dados a cada cinco anos, sendo considerada a maior pesquisa comparativa em Ciências Sociais atualmente.

${ }^{2}$ Duas das exceções são os estudos de Castro, 2000 e Capistrano, 2008.
} 
aplicado a uma distinta realidade sem considerar os seus aspectos particulares. Antes de iniciar a apresentação dos resultados da investigação realizada, essa última ressalva necessita considerações mais detalhadas.

As principais mudanças estruturais encontradas por Inglehart (1990, 1997, 2005) para explicar o impacto da modernização na transformação dos valores das sociedades ocidentais podem ser resumidos nos seguintes pontos: i) aumento da escolaridade; ii) desenvolvimento econômico que acarretaria um aumento da segurança física e econômica dos indivíduos; iii) desenvolvimento científico e tecnológico que trariam maior qualidade de vida por meio do desenvolvimento da medicina, da farmacologia, da nutrição etc. iv) massificação dos meios de comunicação que acarretariam em mudanças no acesso a informação e ao conhecimento. Novamente é necessário ressaltar que tais características da modernização não surgiram e não se consolidaram de forma homogênea em todos os países, especialmente no grupo de países estudados.

Uma ilustração disso é o desenvolvimento econômico desigual observado na segunda metade do século XX se comparados países latinoamericanos com países europeus. Ao selecionar países recorrentemente analisados por Inglehart (Dinamarca, França, Noruega, Suécia, Reino Unido, Argentina, Brasil, Chile, México e Uruguai) observa-se uma considerável diferença entre as regiões. Dados do Banco Mundial $^{3}$ apontam que no ano de 1960 o PIB per capita ${ }^{4}$ médio do grupo europeu era $233 \%$ maior que o latinoamericano, já no ano de 2008 essa diferença aumentou para $344 \%$.

Uma outra dimensão dessa desigualdade é a escolarização. Segundo dados da UNESCO ${ }^{5}$, a evolução na taxa bruta de matrícula nesses países (a razão entre as matrículas registradas na educação regular e o número de habitantes na faixa etária correspondente em cada país para os ensinos primário, secundário e terciário) seguiu padrões distintos de crescimento nas últimas três décadas. No ano de 1975, os países europeus apresentavam uma taxa bruta de matrícula de $75 \%$ e os latinoamericanos de 65\%, no ano de 2007 os primeiros possuíam uma taxa estabilizada de $95 \%$ e grupo da América Latina uma taxa ainda crescente de $86 \%$.

A apreciação desses dois elementos, desenvolvimento econômico e educacional, não é suficiente para questionar a versão da teoria da modernização proposta por Inglehart. Contudo, a observação dos distintos padrões de comportamento dessas mudanças estruturais, permite uma análise crítica do esquema lógico criado pelo autor em relação ao impacto da modernização sobre a cultura política.

${ }^{3}$ World Development Indicators. Disponível em: <http://databank.worldbank.org>. Acesso em: [19 jun. 2009].

4 PIB per capita a preços constantes de 2000 , em US $\$$.

5 UNESCO Institute for Statistics Data Centre. Disponível em: <http://www.uis.unesco.org>. Acesso em: [19 jun. 2009]. 
Interpretações iniciais dessa análise crítica apontariam para a réplica de que o esquema lógico não se altera para os dois grupos de países selecionados, mas apenas identifica uma dinâmica mais acelerada de transformações estruturais em uma determinada região em relação a outra.

De fato, o objetivo da análise crítica colocada não é questionar o esquema lógico proposto. Trata-se de uma alternativa bastante razoável ao problema debatido por Müller e Seligson (1994) sobre a relação causal entre estrutura e cultura política. Contudo, a própria diferença entre a "velocidade" das transformações é um problema fundamental a ser considerado para analisar a relação entre mudanças estruturais e mudança na cultura política.

\section{Teoria social e cultura política}

Identificados os problemas trazidos com as formulações dos conceitos de modernização e cultura política propostos por Inglehart (1990, 1997 e 2005), coloca-se em debate quais as alternativas possíveis para se utilizar o esquema lógico de mudança cultural do autor incorporando questões que são negligenciadas pela má conceituação ou operacionalização dos conceitos.

Uma possibilidade de incorporar o fator histórico, social e da tradição política a essa discussão dentro da cultura política ocorreria por meio de um resgate de fatos históricos importantes de cada sociedade e o estabelecimento de um diálogo entre esses e o estudo de características contemporâneas da sua cultura política. No entanto, uma desvantagem é que tal iniciativa apenas somaria algumas informações, sem enriquecer substancialmente a análise proposta.

Dessa forma, defende-se que as lacunas existentes podem ser preenchidas com mais propriedade por meio de análises alternativas substantivas em relação à formação e o contexto histórico, social e político das diversas sociedades e as interpretações derivadas sobre a modernização. Essas análises podem ser encontradas em trabalhos de teoria social pouco debatidos dentro da ciência política ou subestimados quanto ao seu poder analítico por duas razões principais: primeiramente, a questão da divisão disciplinar que impede o diálogo entre sociólogos, cientistas políticos, historiadores e filósofos políticos; além disso, o sub. campo da cultura política tende a desconsiderar trabalhos que não possuam a empiria como a ênfase do estudo.

De certa forma, tal situação é contraditória visto que o próprio trabalho inicial em que Gabriel Almond propõe o conceito de cultura política: 
"What I propose to do in this brief paper is to suggest how the application of certain sociological and anthropological concepts may facilitate systematic comparison among the major types of political systems..." (ALMOND, 1956, p. 391).

Um diálogo entre o debate presente na teoria social e nos estudos de cultura política pode ser encontrado no resgate de algumas perspectivas de autores latinoamericanos que se tornaram clássicas nas ciências sociais, e alguns estudos de cultura política realizados na região. Sérgio Buarque de Holanda (1971), Raymundo Faoro (1993), Roberto DaMatta (1997), Gino Germani (1981) e Natalio Botana (1994) produziram reconhecidas interpretações da modernização e do impacto social desse fenômeno sobre a cultura política no Brasil e na Argentina.

Em Raízes do Brasil (1995), Sérgio Buarque de Holanda traz à tona uma discussão a respeito da formação social brasileira e a influência da colonização ibérica sobre esse processo. Para Holanda, as relações de dominação estabelecidas desde a chegada dos portugueses acabaram por formar uma sociedade muito desigual, com direitos diferentes, criando na sociedade brasileira o ambiente ideal para a implantação de uma característica marcante da cultura ibérica na sociedade brasileira denominada "personalismo". O conceito de personalismo procura expressar a valorização da individualidade, como decorrência da forte imposição ao desmembramento do coletivo, da contenção, por parte do processo colonizador, de ações coletivistas dentro da sociedade. Para o autor, a origem do personalismo está claramente localizada na península ibérica, pois "Para eles [portugueses e espanhóis], o índice do valor de um homem infere-se, antes de tudo, da extensão em que não precise depender dos demais, em que não necessite de ninguém, em que se baste" (HOLANDA, 1972, p.4).

Para Holanda, essa propriedade específica da cultura ibérica, em que "cada um é filho de si mesmo, de seu esforço próprio, de suas virtudes..." (HOLANDA, 1972, p.5), seria a razão principal de explicação da "frouxidão da estrutura social" e da "falta de hierarquia organizada". Dessa forma, "A falta de coesão em nossa vida social não representa, assim, um fenômeno moderno. E é por isso que erram profundamente aqueles que imaginam na volta à tradição [...] a única defesa possível contra a nossa desordem" (idem).

Em "Os donos do poder" de Raymundo Faoro, o patrimonialismo é uma das principais características da formação política brasileira, influente em todos os grandes episódios desde Dom João I até a era Vargas, e cujo fundamento principal esteve ligado ao problema do poder do Estado sobre a economia. Em concordância com Holanda (1972), Faoro afirma que: 
“O problema não seria pertinente a este ensaio se o feudalismo não houvesse deixado, no seu cortejo funerário, vivo e persistente legado, capaz de prefixar os rumos do Estado moderno. Patrimonial e não feudal o mundo português, cujos ecos soam no mundo brasileiro atual, as relações entre o homem e o poder são de outra feição, bem como de outra índole a natureza da ordem econômica, ainda hoje persistente, obstinadamente persistente" (FAORO, 2001, p.33)

O conceito de capitalismo politicamente orientado, explicativo do surgimento e conservação da ordem patrimolista emerge de apreciação desse caráter da formação polítca brasileira. Nesse ponto, existe uma divergência central em relação ao argumento de Sérgio Buarque de Holanda (1972), pois para Faoro o individualismo, característico de sistemas políticos derivados das revoluções burguesas no fim do feudalismo - ausente na península ibérica e no Brasil, teve de ser suplantado ou incorporado a esse ambiente onde Estado dirigiu o capitalismo:

"O capitalismo politicamente orientado - o capitalismo político, ou o pré-capitalismo - centro da aventura, da conquista e da colonização moldou a realidade estatal, sobrevivendo, e incorporando na sobrevivência o capitalismo moderno, de índole industrial, racional na técnica e fundado na liberdade do indivíduo - liberdade de negociar, de contratar, de gerir a propriedade sob a garantia das instituições. A comunidade política conduz, comanda, supervisiona os negócios, como negócios privados seus, na origem, como negócios públicos depois, em linhas que se demarcam gradualmente. O súdito, a sociedade, se compreendem no âmbito de um aparelhamento a explorar, a manipular, a tosquiar nos casos extremos" (FAORO, 2001, p. 866).

Ao analisar essa obra de Faoro, Paim reconhece os méritos da iniciativa de análise weberiana do patrimonialismo brasileiro, inclusive da herança ibérica destacada por ele, mas considera que "no afã de enfatizar a novidade que trouxe a debate, adotou uma atitude extremamente radical ao deixar de reconhecer o caráter modernizador que o patrimonialismo luso-brasileiro chegou a assumir em certos momentos de sua história" (PAIM, 1998, p. 20).

Para Paim (1998), resgatar o patrimonialismo e suas origens foi fundamental para se analisar a realidade político social brasileira, daí a importância do trabalho de Faoro para a interpretação do sistema político. Contudo, o autor menciona que é importante considerar que esse sistema está exposto a transformações. O caso do ideário modernizante trazido por Marquês de Pombal 
seria uma demonstração exemplar, como afirma o autor, sobre como "a querela do estatismo" poderia ser superada não fosse a forte pressão por desconsiderar essas idéias:

"A passagem de Pombal pelo governo português corresponde a uma autêntica revolução, devotada não à destruição do Estado Patrimonial, e à instauração de regime político plenamente moderno, mas à introdução, em bases duradouras, da componente modernizadora no patrimonialismo tradicional. Essa circunstância de que não se tivesse interessado, simultaneamente, pela modernização das instituições políticas, acrescida do fato de que haja reprimido com ferocidade inquisitorial a tentativa de parte da nobreza de apeá-lo, e a D. José, do poder, levaram a que a historiografia portuguesa optasse por uma linha de combate radical à obra de Pombal..." (PAIM, 1998, p. 37).

Em direção semelhante a Faoro (2001) e Paim (1998), mas com abordagem distinta, DaMatta realiza uma análise de algumas instituições brasileiras. Inspirado na concepção dualista de Louis Dumont de distinção entre sociedades holistas (orientais) e individualistas (ocidentais), o autor aponta que na sociedade brasileira convivem traços holistas e individualistas que tem como base a diferença entre pessoa e indivíduo. Nessa perspectiva há uma combinação entre os elementos do personalismo e do convívio em um sistema patrimonialista de acordo com a adaptação dos atores ao ambiente social:

"É como se tivéssemos duas bases através das quais pensássemos o nosso sistema. No caso das leis gerais e da repressão, seguimos sempre o código burocrático ou a vertente impessoal e universalizante, igualitária, do sistema. Mas no caso das situações concretas, daquelas que a "vida" nos apresenta, seguimos sempre o código das relações e da moralidade pessoal, tomando a vertente do "jeitinho", da "malandragem" e da solidariedade como eixo de ação. Na primeira escolha, nossa unidade é o indivíduo; na segunda, a pessoa. A pessoa merece solidariedade e um tratamento diferencial. O indivíduo, ao contrário, é o sujeito da lei, foco abstrato para quem as regras e a repressão foram feitos" (DAMATTA, 1997, p. 157).

No livro El orden conservador, Botana (1994) analisa a história política argentina no período da virada do século XIX para o século XX. Para o autor, é possível observar nesse período a gênese da rígida separação entre Estado e sociedade e de criação dos mecanismos de perpetuação no poder da elite 
argentina. Essa hegemonia, segundo o autor, foi alcançada principalmente pela aliança entre o governo central e uma maioria de oligarquias provinciais legitimadas pela sociedade.

Embora não seja o seu enfoque principal, Botana não deixa de citar que em seu estudo "estamos en presencia de una línea divisoria de aguas que permite perfilar la trayectoria de un liberalismo argentino e iberoamericano cercano al influjo de la cultura latina en Espana, Francia o Itália" (BOTANA, 1994, p. 9).

A tradição conservadora sobre a qual o autor tece algumas considerações em relação à gênese no início do século $X X$, é a mesma que serve de base para os trabalhos sobre épocas posteriores realizados no Brasil ${ }^{6}$ e na Argentina ${ }^{7}$ sobre a formação do Estado burocrático autoritário e seu papel sobre a manutenção de uma economia liberal em prol da perpetuação de uma elite econômica.

Posteriormente ao livro mencionado, o próprio Botana (1995) argumenta que após 1983 ocorreram mudanças sensíveis na cultura política argentina, principalmente no que se relaciona à sua "herança autoritária". Contudo, afirma que não foi possível anular a tradição e a força que essa herança possui.

Escrevendo sobre um período posterior de intensa industrialização e desenvolvimento econômico na Argentina, nas décadas de 1950 e 1960, Gino Germani (1981) tece diversas considerações sobre o processo de modernização e seu impacto sobre as relações sociais e políticas.

Embora esta seja uma análise muito anterior à realizada por Inglehart e Welzel (2005), existe uma concordância nessa abordagem em relação ao que Germani chama de "requisito universal mínimo" para a existência de uma sociedade moderna e industrial que seria uma "secularização no campo da ciência, tecnologia e economia" (1981, p. 125). Contudo, assim como a preocupação exposta nesse artigo, para o autor "The concern here is not with the great transition prior to the emergence of the first historical instance of industrial society, but with the many transitions produced by the universal diffusion of the new modern-industrial complex" (GERMANI, 1970, p. 155).

Essa preocupação leva Germani a analisar o processo de modernização com enfoque nos casos de países latino americanos, em especial a Argentina. Entre as críticas elaboradas sobre os estudos de modernização da época está uma perspectiva mais cautelosa em relação ao processo de individualização como uma característica incontestável das sociedades industrializadas e modernas:

\footnotetext{
${ }^{6}$ CARDOSO (1993).
}

7 O' DONNEL (1990). 
"There is a tendency to conceptualize the growth of individuality as an evolutionary process. [...] But the process cannot be said to be unilinear, nor can it be conceived of as one of uniform orientations towards higher individuation. Not only may different cultural contexts produce different tyes of processes, in this respect, but even the context of Western culture, there have been various stages of relapse into lower degrees of individuation" (GERMANI, 1981, p. 107).

A revisão dos autores selecionados mostra que as formações políticas e sociais da Argentina e do Brasil possuem elementos muito diversos e mesmo entre os diferentes períodos históricos. Contudo, é possível selecionar aspectos comuns das suas tradições políticas de forma a investigar elementos de influencias sobre a cultura política atual.

Embora não seja um discurso único, e possam ser encontradas diversas divergências entre os autores citados, dois elementos são presentes e se destacam nas falas selecionadas e em diversos outros trabalhos de teoria social. Nessa direção, dois elementos se destacam: i) a influência ibérica sobre os aspectos de formação política e social argentina e brasileira, e ii) a formação do Estado como um ator social central decorrente da tradição e cultura política desses países. Ambos os aspectos são particulares do desenvolvimento político dos países latinoamericanos.

Por outro lado, autores contemporâneos têm selecionado esses comentadores da história social e política argentina e brasileira como fundadores de uma perspectiva cujas interpretações estão sujeitas a contestações. Conforme afirma Souza (2000), ao analisar a perspectiva do iberismo, tais estudos tendem a criar um determinismo histórico, considerando diversos fatores da modernização brasileira e da sua singularidade cultural decorrências de herança ibérica. Dessa forma, consolidou-se no Brasil uma sociologia da inautenticidade baseada em uma equivocada ênfase em elementos pré-modernos para explicação da sociedade.

Schwartzman $(2003,2006)$ também reconhece o tempo e o lugar dessas teorias ligadas a conceitos como o patrimonialismo e o personalismo no Brasil, e propõe uma visão integrada a aspectos mais atuais que poderiam explicar melhor a dinâmica social brasileira. Um exemplo é a mudança na perspectiva em relação ao Estado que poderia explicar não só sua dinâmica e papel como também as origens sociais da oposição ao mesmo. Como pontua o autor: 
"O que podemos observar é que, no Brasil, pelo menos desde 1937, o Estado tem sempre desempenhado um papel ativo e agressivo na implementação de algum tipo de política de desenvolvimento econômico e social, embora fustigado pela crítica liberal antiintervencionista. [...] Desde este ponto de vista, a oposição ao Estado centralizado surge como uma versão retardada do liberalismo econômico do século XIX, florescendo em um enclave mais privilegiado de um país subdesenvolvido, dependente e organizado segundo moldes político-administrativos patrimoniais" (SCHWARTZMAN, 1977, p.177).

Conforme colocam Leiras, Medina e D'Alessandro (2005), Germani e Botana, representaram para os estudos políticos na Argentina uma escola de trabalhos que "produjeron novedosas reconstrucciones de las prácticas, las instituciones, las organizaciones y las ideas políticas durante los siglos XIX y XX."

O conceito de Matriz Sociopolítica (MSP) elaborado por Garretón e outros pode ser considerado um catalisador dessas perspectivas, pois o termo refere-se "à relação entre o Estado, a estrutura de representação ou sistema partidário (combinação de demandas globais e envolvimento político dos atores) e a base socioeconômica dos atores sociais com suas relações e orientações culturais..." (GARRETÓN et al, 2007, p.14).

Segundo Garrentón e seus colegas, o conceito foi criado como uma ferramenta que pudesse organizar aspectos sociais, políticos e culturais, sem desconsiderar a autonomia e interconexão entre esses e, principalmente, sem retirar esses aspectos de seu contexto histórico. Para os autores, a Matriz Sociopolítica latinoamericana pode ser denominada como uma matriz estatalnacional-popular e que possuiria entre seus elementos básicos uma orientação cultural "nacionalista, populista" com atores/sujeitos "orientados primordialmente para o trabalho, a produção, o Estado e a classe política" e, no âmbito do papel do Estado, uma "extensa participação na administração econômica, alta tendência ao estatismo, principal referencial da ação coletiva” (GARRETÓN et al. 2007, p.22).

Usando um questionamento análogo ao problema aqui discutido, seus autores investigam a influência sobre a MSP latinoamericana das principais mudanças estruturais, resumidas em "a construção das democracias políticas; a democratização social e a integração nacional; a reinserção das economias latinoamericanas no sistema mundial; a construção de um modelo de modernidade que assume a globalização e as identidades culturais". (GARRETÓN et al, 2007, p.22). Segundo os autores, resulta dessas transformações um desafio para a MSP latinoamericana que aos poucos sofre transformações substantivas. 
Embora não seja possível esboçar aqui uma genealogia do pensamento culturalista da ciência política e a teoria sociológica latinoamericana para explicar a falta ou propor o diálogo entre as áreas, é possível demonstrar que os estudos de cultura política no Brasil e na Argentina podem se beneficiar de aspectos e contribuições resultantes de outras disciplinas, seja por conteúdo substantivo ou mesmo por "pistas" de processos sociais não explicados sob essa perspectiva.

A maneira com a qual autores como Inglehart enxergam o processo de transformação da cultura política pode ser contraposta ao modelo resultante da avaliação das características da cultura política argentina e brasileira e sua dinâmica discutidas pelos autores ora citados e espelhadas pelo conceito de MSP.

A revisão de teóricos sociais tem por objetivo sofisticar a crítica quanto à relação de causalidade encontrada em alguns casos específicos e a equivocada desconsideração, ou subestimação, de variáveis importantes como a tradição, a história e o contexto social específicos de cada país. Tal argumento fica claro na passagem encontrada em Inglehart e Baker:

"As visões de mundo das sociedades ricas diferem marcantemente das de sociedades pobres. Isto não implica necessariamente em convergência cultural, mas isso prediz a usual direção da mudança cultural e (na medida em que o processo é baseado em uma reposição intergeracional da população), até mesmo, a razão na qual esta mudança é provável de ocorrer" (INGLEHART e BAKER, 2000, p. 23).

A proposta do "renascimento da cultura política" abriu novas possibilidades de estudos por considerar a cultura política não apenas uma variável explicativa fixa, mas também uma variável interveniente. Contudo, essa proposta também apresenta alguns problemas ao tratar a mudança sem considerar o contexto histórico e político particular de cada região e os aspectos distintos de manifestação da cultura política nesses locais.

Após essas considerações é possível darmos início à apreciação de uma dimensão da cultura política que resulta do resgate de algumas perspectivas dos pensadores latinoamericanos, conformada pela tradição e o contexto político e social dessa região. Essa dimensão está ligada ao papel do Estado dentro das relações sociais.

\section{A valorização do Estado na Argentina e no Brasil}

Boa parte do tratamento da importância do Estado decorre da análise do grau de intervenção estatal na economia. Desde a década de $1930 \mathrm{com}$ a Grande depressão, até décadas posteriores ao fim da II Guerra Mundial, o grau de interferência estatal sobre a economia aumentou consideravelmente em vários 
países do Ocidente. Esse aumento se deu tanto pela formação de um Estado comprometido com a garantia do bem-estar social por meio de políticas de diminuição da desigualdade econômica e promoção generalizada da saúde e da educação, quanto pela implementação do Estado desenvolvimentista com vistas ao crescimento da produção nacional.

O advento do Welfare State foi uma das principais causas da mudança de valores (INGLEHART, 1971; 1990; 1998). A partir do momento em que o Estado passou a garantir segurança física e econômica - características raras do período anterior à primeira metade do Século XX - à maioria da população, novos valores passaram a ser preponderantes nessas sociedades.

Além da segurança física e econômica, outra característica bastante marcante, porém não exclusiva do Welfare State, foi o aumento sistemático do nível educacional de determinados países. Os "tigres" do sudoeste asiático são um exemplo emblemático de como a industrialização foi acompanhada de forte expansão da educação primária mesmo sem a implementação do Welfare State. Esta expansão do sistema educacional primário e o aumento no nível educacional demonstraram ser fatores importantes, senão determinantes, para a mudança em direção a valores pós-materialistas (MOORS, 2003).

Por sua vez, a mudança em direção ao pós-materialismo foi, também, a razão do desaparecimento gradual do Welfare State, que acompanhou um movimento de diminuição da intervenção do Estado sobre a economia em diversos países. De acordo com Dezalay e Garth, "A história básica da transformação do Estado na América Latina e nos Estados Unidos da década de 1960 até o ano 2000 pode ser descrita como um deslocamento de Estados desenvolvimentistas ou Estados do BemEstar para Estados neoliberais..." (DEZALAY e GARTH, 2000, p. 163).

Diversos processos foram importantes para que essa transformação pudesse ser realizada. Uma das principais causas está na propagação da ideologia neoliberal que encontrou seu apogeu na implementação do que ficou conhecido como Consenso de Washington. No início da década de 1990, diversas instituições financeiras internacionais endossaram um conjunto de medidas econômicas a serem aplicadas com o objetivo de promover o ajustamento macroeconômico dos países em desenvolvimento que passavam por crises.

Ao comentar esse processo denominado de Consenso de Washington e seu impacto em países latinoamericanos capitaneado pelos bancos credores internacionais, Ferrer afirma que "Toda a estratégia econômica do devedor [economias latinoamericanas] ficava assim submetida ao paradigma central que dessa vez implicava a abertura da economia e a redução do papel do Estado". (FERRER, 2006, p. 241).

Diversas são as razões encontradas pelos estudiosos para explicar o aparente declínio do Estado após a Guerra Fria. Para Ianni (1997), o declínio da 
importância do Estado é causado, principalmente, pelo surgimento de novos atores que assumem papéis antes exclusivos do Estado.

Segundo Fukuyama $(1997,2005)$, as perspectivas e orientações em relação ao Estado na segunda metade do Século XX, foram fortemente influenciados pelo processo de modernização e de disseminação dos valores liberais. Tais processos, que não encontram rivais na atualidade, estariam produzindo uma posição estabelecida na população sobre o relacionamento ideal entre economia e Estado em prol de uma autonomia maior dos atores econômicos.

Para Kwasnicki, o processo de aumento ou diminuição da intervenção estatal está ligado à "aceitação social" que as idéias estatistas ou liberais recebem ao longo do tempo nas diversas sociedades:

"The way of changes of social attitudes toward two opposing views of economic development, namely statism and liberalism, in the last 300 hundred years in the Western societies is suggested. Domination of any one of these opposing views of social development in different periods is never total. At any time there are people claiming that the right way of social development is opposing to currently prevailing mode of development. This opposing view allows to initiate the issuing substitution phase what occurs when conditions of socioeconomic development are worsening" (KWASNICKI, 2000, p.4).

Em um estudo comparativo entre o Japão e os Estados Unidos, Lipset discute essa questão como uma dimensão decorrente de formações culturais distintas. Para Lipset, “...variations in political orientation and social policies between Japan and America [United States] are linked to basic differences in orientation toward individualism and equality..." (LIPSET, 1994, p. 194). Após seu estudo que envolveu pesquisas de opinião pública nos dois países, o autor chega à conclusão de que embora haja uma discussão forte em torno da convergência de opiniões e atitudes que legitimem um tipo de regime e política do Estado, as diferenças culturais são significativas nesse processo: 
"The two nations follow different organizing principles. National traditions continue to inform the cultures, economies and politics of both countries in very dissimilar ways. One, the United States, follows the individualistic essence of bourgeois liberalism and evangelical sectarian Christianity; the other, Japan, reflects the group orientated norms of the post-feudal aristocratic Meiji era. The former still stresses equal respect across stratification lines; the latter still emphasizes hierarchy in interpersonal relations. The first continues to suspect the state; the second places heavy reliance on its directing role" (LIPSET, 1994, p.196).

De forma semelhante, Svampa analisa a influência dessa nova conformação do Estado sobre aspectos da cultura política argentina:

"el cambio en las formas de regulación estatal, la primacía del mercado como mecanismo de inclusión, van de la mano de una concepción del individuo (o del proceso de individualización), que enfatizan la noción de sujeto competente, de autorregulación, esto es, un individuo al cual se exige que se haga cargo de sí y que desarrolle las competencias y recursos necesarios para su inclusión en el mercado y el acceso a los bienes sociales, independiente de los recursos y soportes pre-existentes. Claro está, no es lo mismo que esta exigencia de individualización o autoregulación en el marco de un estado de bienestar, que en medio de un proceso de desregulación vertiginoso y radical, como el que se operó en Argentina ... Hay una frase de un sociólogo italiano, Giussepe Améndola, quien dijo, "se autorregulan los que pueden, pero para los que no pueden está el Estado" (SVAMPA, 2004, p.9 . 10).

Baquero, por sua vez, analisa a aparente contradição entre o apoio aos regimes neopopulistas na América Latina, que deram suporte ao neoliberalismo, e o descontentamento da opinião pública em relação às reformas neoliberais: 
"neste contexto, o tipo de cultura política que emerge com a institucionalização do neoliberalismo se caracteriza pelo distanciamento que se estabelece entre a sociedade e o Estado, restringindo a participação popular exclusivamente pelo voto (...) 0 neopopulismo, portanto, não só consegue sobreviver mas se institucionaliza como arma para garantir legitimidade aos governos neoliberais. Ao mesmo tempo, contribui para institucionalização de práticas políticas individuais em detrimento de ações coletivas" (BAQUERO, 1996 p.138).

Em estudo a partir dos dados da pesquisa World Values Survey, Castro verificou que os brasileiros possuíam então um maior apego aos valores chamados neoliberais que os argentinos. Considerando que as reformas econômicas neoliberais ocorreram antes na Argentina que no Brasil, o autor considera que uma das explicações possíveis para a diferença seria o tempo. Em suas palavras,

"ao experimentar as mudanças neoliberais e a retirada do Estado da área econômica e social sem que tenha havido um substitutivo à altura, as pessoas tenderiam a se colocar contra as reformas. A substituição do Estado pelo mercado não foi acompanhada de um aumento de renda que compensasse a sua retirada de esfera social. Desta forma, passada a euforia e a fase épica, a realidade provavelmente não propiciou a melhoria de vida que as mudanças econômicas prometeram" (CASTRO, 2000, p.172).

Em uma interpretação mais recente, após analisar alguns resultados mais atualizados da pesquisa de cultura política Latinobarômetro para a Argentina, Vitullo parece corroborar os estudos de Castro, e verifica uma forte queda no apoio às privatizações e aumento nos índices de suporte ao gerenciamento estatal de diversas áreas. Para o autor, "Essas opiniões se inscrevem numa crescente onda de descontentamento frente às políticas de cunho neo-liberal, onda que vem se expandindo, com distintos graus de intensidade pelo conjunto do continente nesses últimos anos" (VITULLO, 2007, p. 97).

Essa posição traz à tona uma reação recente da opinião pública em relação às reformas econômicas ocorridas em quase todos os países latinoamericanos durante a década de 1990 que tiveram impacto direto sobre a cultura política.

Para Inglehart, "the widely prevailing view among psychologists that traits such as individualism rather than collectivism are fixed traits of given cultures should be modified: they do indeed reflect given cultural heritage to some extent, the emergence, but the emphasis can change over time..." (INGLEHART, 2005, p.144) e essa mudança é a principal característica das sociedades pós-industriais ocidentais, processo que 
se verifica por meio do "desenvolvimento humano". Segundo o autor, a tendência do "desenvolvimento humano" que envolve uma série de mudanças de prioridades individuais calcadas no aumento do individualismo, da auto-expressão, da autonomia individual, é o aspecto central da transformação da cultura política na virada do século XX ao século XXI.

Almeida também identifica uma síndrome semelhante no Brasil, que estaria relacionada ao aumento de escolaridade. Para o autor, a escolaridade é o fator fundamental de determinação da cultura política estatista: "Para quem deseja menos Estado, resta apenas o consolo de que esse é o destino da opinião pública no Brasil. É um processo longo, porém inexorável: também nesse aspecto, à medida que a escolaridade aumentar, o apoio social à presença do Estado na economia tenderá a tornar-se cada vez menor" (ALMEIDA, 2007, p. 193). (...) "Para mudar essa mentalidade hierárquica e próestatal é preciso escolarizar a população" (Idem, p. 210).

Embora trate dessa questão de forma indireta, Castro enxerga na expressão da cultura política em relação às reformas estruturais da economia e do Estado uma posição coerente em relação à tradição política de países como Argentina, Brasil e Chile. Outra característica deste final de milênio é a exaltação do individualismo:

"A máxima romana Homo Homini Lupus, revivida por Hobbes no século XVI, parece que foi cunhada para os tempos atuais. Sabe-se que o individualismo é considerado uma das bases da formação do capitalismo na Inglaterra e que se constituiu em uma das mais fortes formas de expressão das sociedades capitalistas avançadas. No entanto, essa não é a nossa tradição. A base de formação da nossa sociedade seguiu outra via, que tem no Estado a sua origem e força [...] Assim, ao serem analisadas as relações entre democracia e mudanças econômicas no três países, salienta-se que o padrão de uma cultura política fragmentada e individualista tem conformado as atitudes das pessoas. Desta forma, o apoio às mudanças neoliberais é completamente congruente com a formação histórica dos países" (CASTRO, 2000, 154-155).

O World Values Survey possui uma bateria de questões que buscam identificar posicionamento em relação ao Estado e ao mercado. Nessa bateria o entrevistado é estimulado a se posicionar em uma escala de 1 a 10 em que os pontos extremos representam às posições expostas na Figura 1. 


\section{Figura 1}

\section{Bateria de itens Mercado vs. Estado}

\begin{tabular}{|c|l|l|}
\hline $\mathbf{v 1 1 7}$ & $\begin{array}{l}\text { Empresas e negócios particulares } \\
\text { devem aumentar }\end{array}$ & $\begin{array}{l}\text { Empresas e negócios do governo } \\
\text { devem aumentar }\end{array}$ \\
\hline $\mathbf{V} 118$ & $\begin{array}{l}\text { As pessoas deveriam se } \\
\text { responsabilizar mais por elas } \\
\text { mesmas }\end{array}$ & $\begin{array}{l}\text { O governo deveria se responsabilizar } \\
\text { mais para garantir boas condições de } \\
\text { vida a todos }\end{array}$ \\
\hline $\mathbf{V} 119$ & $\begin{array}{l}\text { A concorrência é boa porque estimula } \\
\text { as pessoas a trabalhar } \\
\text { mais e desenvolver novas idéias }\end{array}$ & $\begin{array}{l}\text { A concorrência é ruim porque } \\
\text { desperta o que há de pior nas } \\
\text { pessoas }\end{array}$ \\
\hline $\mathbf{V} 121$ & $\begin{array}{l}\text { No futuro, a pessoa trabalhadora } \\
\text { consegue uma vida melhor. } \\
\text { custas dos outros }\end{array}$ & $\begin{array}{l}\text { Ser uma pessoa trabalhadora } \\
\text { não necessariamente traz sucesso. É } \\
\text { mais uma questão de sorte e de } \\
\text { contatos }\end{array}$ \\
\hline
\end{tabular}

Fonte: Questionário WVS (2006) - Versão português, Brasil.

Em uma análise sobre essa bateria de questões, Andrews (2006) avalia a consistência interna dos itens mencionados com vistas à possibilidade de identificar um padrão de legitimação do setor privado frente ao setor público. Após uma análise de correlações entre as variáveis da bateria. Andrews conclui que, “de um modo geral, os resultados indicam que as quatro variáveis do WVS carecem de uma clara consistência interna. Assim sendo, não devem ser considerados como uma única dimensão, capaz de identificar a existência de uma "síndrome de cultura política" que se refira ao setor público e ao setor privado" (ANDREWS, 2006, p. 4).

Deve ser destacado, contudo, que a incongruência dos resultados levantados pela autora deve-se à sua perspectiva de análise das questões do WVS para medir a tipologia dos "três mundos" de Esping.Andersen. A contestação do nível macro e micro das variáveis é embasada na incongruência dos resultados à luz dessa perspectiva teórica específica.

O teste mais aprofundado da consistência interna das questões utilizadas nessa bateria foi realizado por meio da análise fatorial desses itens para cada onda. Os resultados dessa análise estão expostos na Tabela 1. 
Tabela 1

Análise fatorial de questões sobre Mercado X Estado no WVS (1991, 1995-1997 e 2006)

\begin{tabular}{|c|c|c|c|c|c|c|}
\hline Itens & \multicolumn{2}{|c|}{ Argentina (2) } & \multicolumn{2}{|c|}{ Argentina (3) } & \multicolumn{2}{|c|}{ Argentina (5) } \\
\hline & 1 & 2 & 1 & 2 & 1 & 2 \\
\hline Igualdade de renda & $-0,466$ & 0,132 & $-0,679$ & 0,082 & 0,002 & 0,799 \\
\hline $\begin{array}{l}\text { Propriedade do governo } \\
\text { ou privada }\end{array}$ & 0,739 & 0,016 & 0,660 & 0,081 & 0,269 & $-0,423$ \\
\hline $\begin{array}{l}\text { Resp. do governo ou } \\
\text { individuo }\end{array}$ & 0,683 & $-0,079$ & 0,493 & 0,150 & 0,232 & 0,669 \\
\hline Competição boa ou ruim & 0,630 & 0,255 & 0,409 & 0,523 & 0,793 & $-0,098$ \\
\hline Trabalho leva ao sucesso & 0,174 & 0,768 & 0,165 & 0,689 & 0,711 & 0,190 \\
\hline Acumulação de riqueza & 0,224 & $-0,711$ & 0,153 & $-0,746$ & $-0,371$ & 0,019 \\
\hline Itens & $\mathrm{Bra}$ & & $\mathrm{Bra}$ & (3) & $\mathrm{Bra}$ & (5) \\
\hline & 1 & 2 & 1 & 2 & 1 & 2 \\
\hline Igualdade de renda & $-0,020$ & 0,621 & 0,723 & 0,056 & $-0,138$ & 0,794 \\
\hline $\begin{array}{l}\text { Propriedade do governo } \\
\text { ou privada }\end{array}$ & 0,677 & 0,011 & $-0,003$ & 0,699 & 0,085 & 0,624 \\
\hline $\begin{array}{l}\text { Resp. do governo ou } \\
\text { individuo }\end{array}$ & 0,718 & 0,020 & $-0,595$ & 0,203 & 0,380 & 0,375 \\
\hline Competição boa ou ruim & 0,698 & 0,00 & $-0,080$ & 0,639 & 0,736 & 0,109 \\
\hline Trabalho leva ao sucesso & 0,327 & 0,517 & 0,546 & 0,189 & 0,652 & 0,004 \\
\hline Acumulação de riqueza & $-0,110$ & 0,677 & $-0,084$ & $-0,330$ & $-0,408$ & 0,038 \\
\hline
\end{tabular}

Nota: a) Rotação varimax.

b) $\mathrm{N}_{\text {Arg (2) }}=892 ; \mathrm{N}_{\text {Arg (3) }}=1011 ; \mathrm{N}_{\text {Arg (5) }}=896 ; \mathrm{N}_{\text {Bra (2) }}=1676 ; \mathrm{N}_{\text {Bra (3) }}=1098 ; \mathrm{N}_{\text {Bra (5) }}=1472$

c) \% variação explicada pelos pares de componentes se mantiveram entre $40 \%$ e $50 \%$.

d) (2) Onda referente ao ano de 1991, (3) anos de 1995-1997 e (5) ano de 2006

Fonte: European and World Values Survey (XWVSEVS_1981_2005_v20080220.sav)

Em um primeiro exame, é possível afirmar que os modelos apresentam um razoável percentual de explicação (entre $40 \%$ e 50\%) visto que o mesmo apresenta um alto grau de simplicidade.

Além disso, a análise demonstra que, para alguns itens, os altos coeficientes de alguns fatores no mesmo componente, demonstram certa coerência entre as questões "Propriedade do governo ou privada" com "Responsabilidade do governo"; e "Trabalho leva ao sucesso" com "Acumulação de riqueza". Devido ao destaque nos coeficientes do primeiro par em uma mesma dimensão, é razoável destacar essas duas variáveis como as mais adequadas para se trabalhar a dimensão selecionada. 
O Gráfico 1 aponta a distribuição do item "Responsabilidade do Governo" ao longo das sucessivas aplicações na Argentina e no Brasil. Os valores mais altos representam uma posição mais próxima da opinião de que "O governo deveria se responsabilizar mais para garantir boas condições de vida a todos".

\section{Gráfico 1}

\section{Boxplot de Responsabilidade do governo por país}

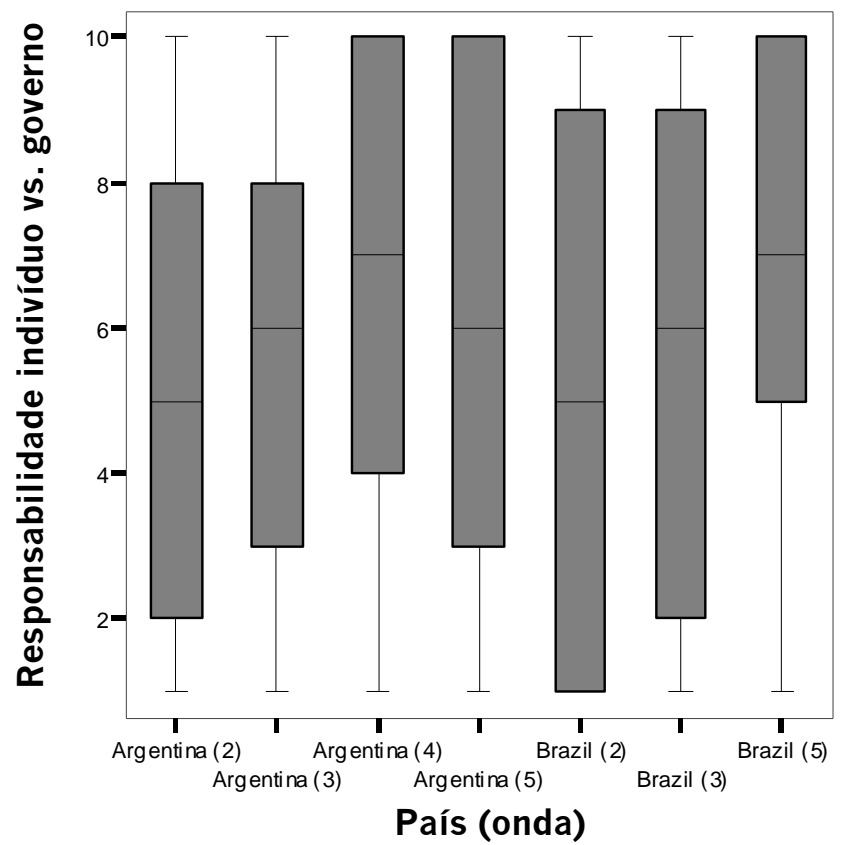

Nota: (2) Onda referente ao ano de 1991, (3) anos de 1995-1997, (4) ano de 1999 e (5) ano de 2006 Fonte: European and World Values Survey (XWVSEVS_1981_2005_v20080220.sav)

Assim como na questão sobre responsabilidade do governo, o crescimento da proporção daqueles que preferem o aumento de empresas e negócios do Estado, como aponta o Gráfico 2, pode ser uma indicação de que o "estatismo" seja crescente ao longo do tempo. No entanto, se faz necessária uma análise mais aprofundada da relação dessa variável da cultura política com as mudanças estruturais. 


\section{Gráfico 2 \\ Boxplot de Propriedade do governo ou privada por país}

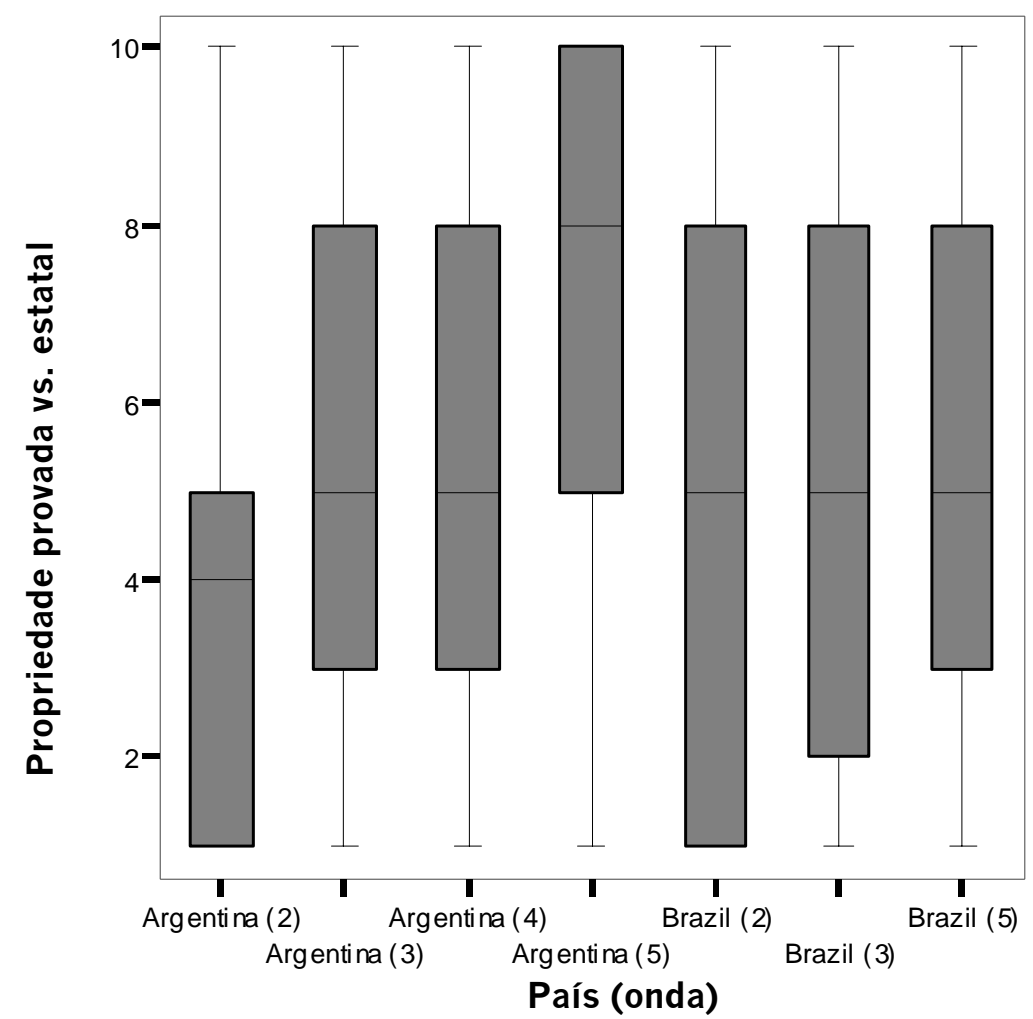

Nota: (2) Onda referente ao ano de 1991, (3) anos de 1995.1997, (4) ano de 1999 e (5) ano de 2006 Fonte: European and World Values Survey (XWVSEVS_1981_2005_v20080220.sav)

Uma forma de se tentar captar a mudança intergeracional é por meio da análise de coorte. As coortes foram criadas em faixas de idade de 5 em 5 anos, de modo que uma faixa de idade entre "25 e 29 anos", seja analisada como o mesmo grupo de indivíduos 5 anos mais velhos com "30 a 34 anos". Nos Gráficos 3 e 4, a mesma distribuição da questão sobre empresas e negócios do governo foi analisada mas segundo as coortes. As coortes mais jovens estão à esquerda e as coortes mais velhas à direita. 
Gráfico 3

Boxplot de Propriedade do governo ou privada por coorte Argentina

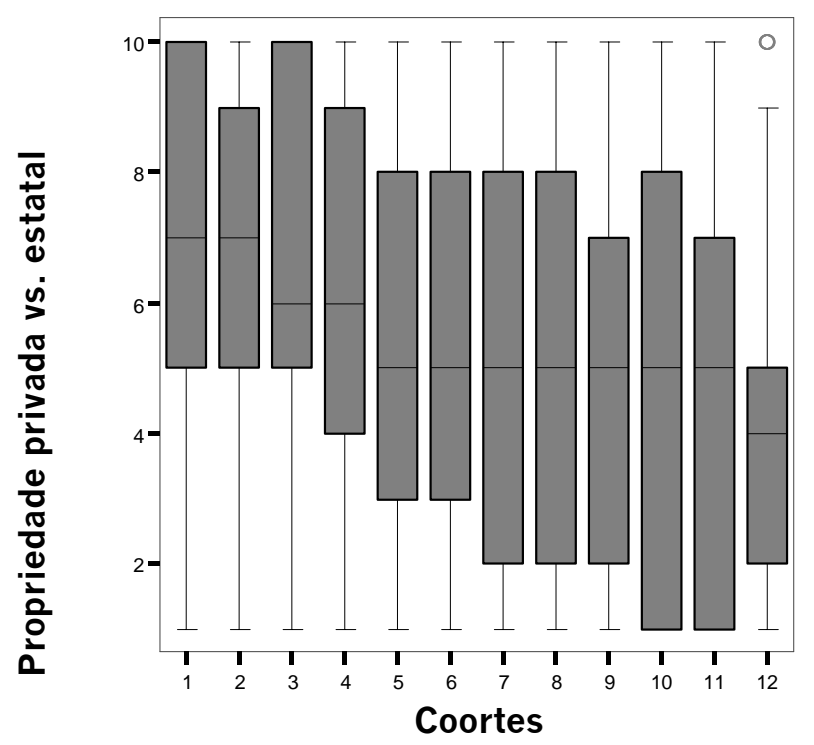

Gráfico 4

Boxplot de Propriedade do governo ou privada por coorte Brasil

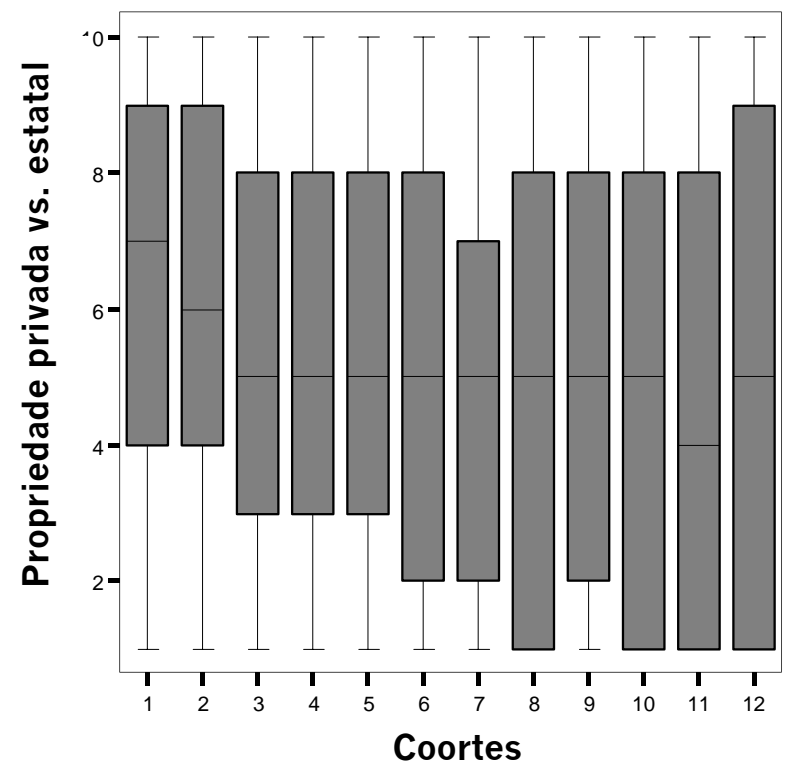

Fonte: European and World Values Survey (XWVSEVS_1981_2005_v20080220.sav) 
Esses resultados trazem uma visão mais clara da tendência que essa questão pode apresentar. As coortes mais jovens na Argentina são significativamente mais próximas da posição de que os negócios e empresas do governo devem aumentar. Embora o Brasil não apresente o mesmo grau de diferença entre as coortes, também é possível afirmar que as gerações mais novas tendem a se aproximar da posição estatista.

Essa questão pode refletir, de certa forma, a insatisfação apontada pelas populações de Argentina e Brasil com o processo de privatização de diversos serviços públicos ocorrido durante a década de 1990 (ANDREWS, 2006; Relatório Latinobarômetro, 2005). Contudo, é importante notar que essa é uma tendência verificada ao longo de três décadas com períodos anteriores e posteriores a esse processo.

A partir desses resultados, um questionamento a ser realizado é sobre o que determina essa predominância do perfil "menos estatal" ou "mais liberal": o nível de escolaridade ou o ambiente social, os valores, as orientações e atitudes historicamente compartilhadas por essa classe de renda mais elevada?

A resposta pode emergir da análise agregada de todos esses grupos. Embora as mudanças estruturais apontem para o aumento de escolaridade, o aumento na renda per capita e na qualidade de vida, para a "trajetória do desenvolvimento humano", nos termos de Inglehart e Welzel (2005), esses aspectos se confrontam com uma dimensão que tende a perdurar na cultura política de Brasil e Argentina: a legitimação da autoridade do Estado.

Embora não seja possível observar claramente a tendência ao individualismo ou à autonomia individual nesses dois países durante o período avaliado pelo WVS, também não é possível identificar uma tendência de rejeição do Estado como foi exposto sendo que essas duas dimensões não são necessariamente opostas como seria previsto pela hipótese de Inglehart.

\section{Considerações finais}

Após as décadas de liberalização econômica, esse início de século se caracteriza por uma legitimação crescente da ação do Estado por parte da opinião pública na Argentina e no Brasil. Essa situação se deve em parte às crises e reformas econômicas das décadas de 1980 e 1990, mas também tem relação com a tradição da cultura política latinoamericana de matriz estatista.

Essa constatação não contradiz a tendência ao individualismo observada por Inglehart e Welzel (2005). Como propôs Catterberg (1991), as duas dimensões "individualista" e "estatista" da cultura política convivem no caso argentino, e como demonstrou-se nessa análise, não é possível afirmar que as duas tendências são opostas para as sociedades argentina e brasileira. 
Uma diferenciação entre os conceitos de autonomia e de individualismo sofisticaria o próprio conceito de modernização que para alguns, com o crescente individualismo, tenderia a um cenário de estado natural hobbesiano de todos contra todos, que daria margem à instauração de regimes autoritários. Não é essa também a tendência com o aumento do estatismo. O apoio a uma ação mais incisiva da autoridade do Estado não está diretamente ligada a um apoio ao retorno de um regime autoritário.

Como foi apontado, o conceito de cultura política necessita ser revisto para dar conta da extrema diversidade entre culturas, histórias políticas e contextos sociais. Não se propõe aqui a revisão das bases conceituais de Almond e Verba (1963), mas uma atenção redobrada aos estudos comparativos que utilizam um modelo determinado e fixo de cultura política que podem, como foi apontado, apresentar forte viés etnocêntrico.

Dessa forma, propõe-se desenvolver novos estudos e pesquisas sobre a cultura política na América Latina (seus fundamentos, sua constituição, seu papel, sua função nas sociedades latinoamericanas e sua relação com a estrutura política) que dêem conta não somente de sua realidade específica, mas que vá além da mera extensão de estudos ou da aplicação de modelos formulados com base em outras realidades sociais, culturais, econômicas e políticas. Ou seja,

“... um estudo que vise a ir além da aceitação do modelo de cultura cívica deve partir dos conceitos conhecidos, dos trabalhos teóricos e empíricos realizados e dos instrumentos metodológicos disponíveis para procurar construir uma teoria de cultura política adequada às condições da América Latina (CASTRO, 2008, p. 23)."

A proposta de renascimento da cultura política levada a cabo por Ronald Inglehart (1988), entre outros, já logrou êxito visto a consolidação desse sub-campo de estudos da Ciência Política. Suas teses sobre a mudança na cultura política são fundamentais para se compreender a realidade política de diversos países do mundo hoje. No entanto, devido ao escopo de sua teoria, diversos aspectos de seus estudos demandam um aprofundamento teórico e empírico por meio de testes mais focados em culturas políticas específicas.

Como foi exposto neste artigo, alguns aspectos da tradição política presentes em debates dentro da teoria social latinoamericana e ausentes do mainstream da ciência política são essenciais para se compreender os mais variados elementos da cultura política, como o apoio ao Estado, por exemplo. Além disso, se faz necessária uma análise mais extensa que inclua outros países da América Latina. Como aponta a Figura 2, existem evidências de que o padrão identificado na Argentina e no Brasil pode estar presente em demais países dessa região. 
Figura 2

\section{"O Estado pode solucionar problemas" - América Latina \\ Latinobarômetro 1998-2007}

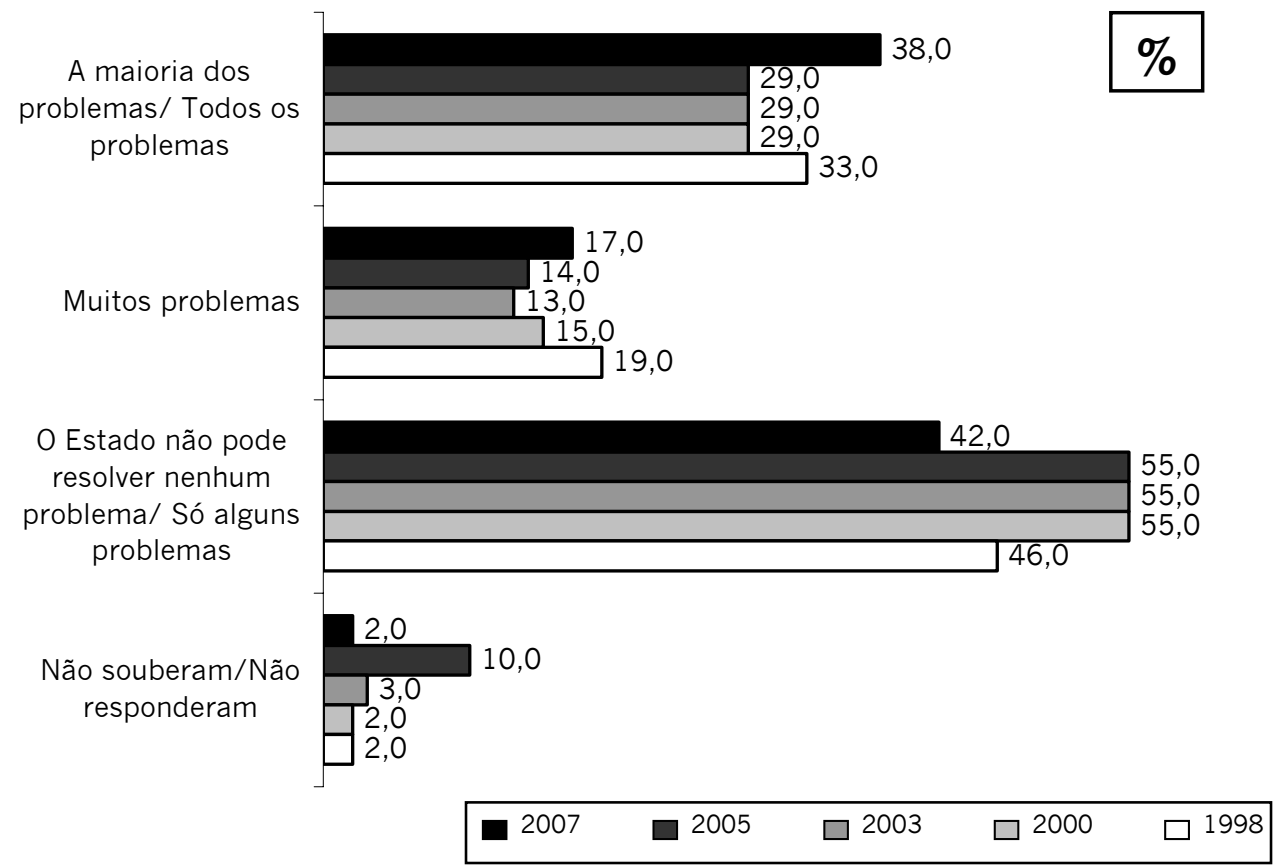

Pergunta: Se dice que el Estado puede resolver los problemas de nuestra sociedad porque tiene los médios para ello. ¿Diria usted que el Estado puede resolver todos los problemas, la mayoría de los problemas, bastantes problemas, sólo algunos problemas o el Estado no puede resolver ningún problema

Fonte: Informe Latinobarômetro, 2007. Disponível em: <http://www.latinobarometro.org/>. Acesso em: [out. de 2008].

Por fim, os resultados aqui apontados não foram explorados de maneira suficiente para uma interpretação mais sofisticada da relação entre cultura política e desempenho do regime político dado o próprio objetivo do estudo. No entanto, os aspectos aqui levantados da cultura política no Brasil e na Argentina são relevantes para se compreender o atual quadro político latinoamericano. O crescente surgimento de líderes chamados de neo-populistas, a discussão sobre a reforma do Estado, o constante debate entre auto-regulação do mercado e intervenção do Estado, o impacto das crises econômicas e políticas, entre outros, são discussões que carecem de reflexões como as propostas neste artigo. 


\section{Referências Bibliográficas}

ALMOND, G. "Comparative political systems". Journal of Politics, Cambridge: Cambridge University Press, 18, p.391-409, 1956.

. "The Intellectual History of the Civic Culture Concept". In: ALMOND, G.; VERBA, S. The Civic culture revisited. Newbury park: Sage, 1980.

ALMOND, G. e VERBA, S. The civic culture. Princeton: Princeton University Press, 1963.

ANDREWS, C. W. "Investigando a legitimidade: estudo de atitudes de público em relação aos papéis do Estado e do mercado." Opinião Pública, Campinas. Vol.12, nº 2, p. 407.431, nov. 2006.

DEVOTO, F; FAUSTO, B. Argentina - Brasil: 1850-2000. Un ensayo de historia comparada. Buenos Aires: Sudamericana, 2008.

BAQUERO, M. Cultura política e democracia: Os desafios das sociedades contemporâneas. Porto alegre: UFRGS, 1994.

Cultura Política e Neoliberalismo na América Latina. In: PINTO, C. R; GUERREIRO, H. (org.). América Latina: o Desafio da Democracia nos Anos 90. Porto Alegre: Ed. UFRGS/Grupo Montevideo, 1996.

Democracia e desigualdades na América Latina - Novas perspectivas. Porto Alegre: Ed. UFRGS, 2007.

BOTANA, N. El orden conservador: la política argentina entre 1880 y 1916 . (Editorial Sudamericana). Buenos Aires: 1994.

Las transformaciones institucionales en los años del menemismo En: SIDICARO, R. y MAYER, J. (comp.). Política y Sociedad en los años del menemismo. Buenos Aires: Oficina de Publicaciones del CBC, 1995.

CARDOSO, F. H. A construção da democracia: estudos sobre política. São Paulo: Siciliano, 1993.

CASTRO, H. C. O. "Cultura política: a tentativa de construção de um conceito adequado à América Latina." Revista de Estudos e Pesquisas sobre as Américas, vol. 2, $\mathrm{n}^{\circ} 1$, jan-jun. p.03. 2008 [Online]. Disponível em:

<http://www.repam.org/edicoes/02/index.html> Acesso em: [10 abr. 2009]. 
Democracia e mudanças econômicas no Brasil, Argentina e Chile: um estudo comparativo de cultura política. Porto Alegre. [Tese (doutorado)], Programa de Pós. Graduação em Ciência Política, Universidade Federal do Rio Grande do Sul, 2000.

CASTRO, H. C. O. ; CAPISTRANO, D. "Cultura Política Pós-Consenso de Washington: o conceito de Cultura Cívica e a mudança política na América Latina." Revista Debates. UFRGS, vol. 2, p.75-97, 2008.

CAPISTRANO, D. A tese do pós-materialismo e o caso latinoamericano. Brasília, Trabalho de Conclusão de Curso (Graduação em Sociologia). Departamento de Sociologia - UnB, 2006.

- Argentinos e brasileiros frente ao Estado: um estudo de cultura política comparada. Brasília. Dissertação (mestrado) - Universidade de Brasília, Instituto de Ciências Sociais, Centro de Pesquisa e Pós-Graduação sobre as Américas, Programa de Mestrado Estudos Comparados sobre as Américas, 2008.

CATTERBERG, E. Argentina confronts politics: political culture and public opinion in the Argentine transition to democracy. London: Lynne, 1991.

DAMATTA, R. Carnavais, Malandros e Heróis: Para uma sociologia do dilema brasileiro. Rio de Janeiro: Ed. Rocco, 1997.

FAORO, R. Os donos do poder. 3a. Edição. Rio de Janeiro: Editora Globo, 2001. "A aventura liberal numa ordem patrimonialista." Revista USP, São Paulo, n¹7, mar./abr. 1993.

FUKUYAMA, F. O fim da história e o último homem. Rio de Janeiro: Rocco, 1992 . Construção de Estados: Governo e organização no século XXI. Rio de Janeiro: Rocco, 2005.

GARRETÓN, M. A. et al. América Latina no Século XXI. Em Direção A Uma Nova Matriz Sociopolítica. Rio de Janeiro: Editora FGV, 2007.

GERMANI, G. The Sociology of Modernization: Studies on Its Historical and Theoretical Aspects with Special Regard to the Latin American Case. New Brunswick: Transaction Books, 1981.

HOLANDA, S. B. Raízes do Brasil. 6a ed. Rio de Janeiro: Editora José Olympio, 1971. 
IANNI, O. Teorias da Globalização. Rio de Janeiro: Civilização Brasileira, 1997.

INGLEHART, R. Cultural shift in advanced industrial society. Princeton-NJ: Princeton University Press, 1990.

. Modernización y postmodernización: El cambio cultural, económico y político en 43 sociedades. Madrid: CIS/Siglo XXI, 1998.

INGLEHART, R. e BAKER, W. "Modernization, cultural change and the persistence of traditional value". American Sociological Review. vol. 65, p.19-65, 2000.

INGLEHART, R. e CATTERBERG, G. "Trends in Political Action: The Developmental Trend and the Post-Honeymoon Decline". International Journal of Comparative Sociology, Michigan, 43, p.300-316, 2002.

INGLEHART, R. e WELZEL, C. "Democratic Institutions and Political Culture: Misconceptions in Addressing the Ecological Fallacy." Comparative Politics 35(4), p.61-79, 2003.

Modernization, Cultural Change and Democracy. Cambridge: Cambridge University Press, 2005.

KWASNICKI, W. "Centennial Waves of Socio-Economic Development (SocioEconomic Development and Transformation in a Long-Term Perspective)" [Online]. Paper presented at The Eighth International Joseph A. Schumpeter Society Conference - The Millennium Conference: Manchester/UK. 2000 Disponível em: <http://prawo.uni.wroc.pl/ kwasnicki/>. Acesso em: [02 jul.2008].

MOISÉS, J. A. Os Brasileiros e a Democracia: Bases sócio-políticas da legitimidade democrática. São Paulo: Ática, 1995.

MULLER, E. N; SELIGSON, M. A. "Civic Culture and Democracy: The Question of Causal Relationships." American Political Science Review, vol. 88, n 3, 1994.

O`DONNEL, G. Análise do Autoritarismo Burocrático. Rio de Janeiro: Paz e Terra, 1990.

SOUZA, J. A modernização seletiva: uma reinterpretação do dilema brasileiro. Brasília: Editora UnB, 2000. 
SCHWARTZMAN, S. "As Eleições e o Problema Institucional (1): Estado e Sociedade no Brasil." Dados - Revista de Ciências Sociais, Rio de Janeiro, v. 14 . p.164 - 184. 1977.

. "Atualidade de Raymundo Faoro". DADOS - Revista de Ciências Sociais, Rio de Janeiro, vol. 46, $n^{\circ}$ 2, p.207-213, 2003.

- Nota sobre o patrimonialismo e a dimensão publica na formação da América Latina contemporânea. [Online], 2006. Disponível em:

$<$ www.schwartzman.org.br/simon/patrimonialismo06.pdf>. Acesso em: [26 out.2007].

SVAMPA, M. "Cinco tesis sobre la nueva matriz popular." Lavboratorio, Revista de estudios sobre cambio social, [online] Instituto G.Germani, Fac. De Ciencias Sociales, Universidad de Buenos Aires. Año IV, número 15, primavera. Buenos Aires, 2004.

VITULLO, G. Teorias da democratização e democracia na Argentina contemporânea. Porto Alegre: Sulina (coedição UFRN), p.2007.

Daniel Jaime Capistrano - capistrano@unb.br

Henrique Carlos de O. Castro - henrique@unb.br

Recebido para publicação em julho de 2009. Aprovado para publicação em fevereiro de 2010. 